\title{
Detecting vortical structures in time-resolved volumetric flow fields
}

\author{
Karuna Agarwal1', Omri Ram¹, Jin Wang1, Yuhui Lu¹, Joseph Katz ${ }^{1 *}$ \\ ${ }^{1}$ Johns Hopkins University, Department of Mechanical Engineering, Baltimore, USA \\ *katz@jhu.edu
}

\begin{abstract}
The detection of three-dimensional coherent vortical structures that get advected as well as deformed with time is a challenge. However, it is critical for the statistical analysis of these vortices, for example, the quasi-streamwise vortices (QSVs) in the near field of a turbulent shear layer, where cavitation inception typically occurs. These structures exhibit underlying correlations among different properties that can be derived from the velocity gradients. Exploiting these correlations, a pseudoLagrangian vortex detection method is proposed that uses $k$-means clustering based on vorticity magnitude and direction, values of $\lambda_{2}$, strain rate structure, axial stretching, and location. The method facilitates the finding that QSVs have pressure minima that are lower than those in the surrounding flow, including the primary spanwise vortices. These minima typically appear after a period of axial stretching and before contraction events.
\end{abstract}

\section{Introduction}

The evolution of vortical structures is of interest in a plethora of turbulent flows since they play major roles in momentum and energy transport, interactions with boundaries, and are primary sites for cavitation inception. Hence, many previous studies have attempted to identify them based on several criteria e.g., the second velocity gradient invariant or the Q-criterion (Hunt et al., 1998), the intermediate eigenvalue $\left(\lambda_{2}\right)$ of the sum of squares of symmetric and asymmetric parts of the velocity gradient tensor (Jeong and Hussain, 1995), and swirling strength (Adrian et al., 2000). These Eulerian methods are localized in space and require selection of appropriate thresholds. Hence, they might not be continuous in time owing to e.g. experimental errors. Lagrangian techniques that are based on the trajectories of fluid particles, such as Finite Time Lyapunov Exponents (Haller and Sapsis, 2011) or coherent structure coloring (Schlueter-Kuck and Dabiri, 2017), have also been implemented for structures that are relatively low-ranked in space and time. The detection of complex evolving threedimensional structures has remained a challenge.

With the advent of time-resolved tomographic particle tracking involving Shake-the-Box (STB, Schanz et al., 2016) method and subsequent pressure calculations (Wang et al., 2019), it is possible to measure the 3D evolution of vortices in turbulent flows. In this study, these methods are employed to characterize the evolution of intermittent quasi-streamwise vortices (QSVs) that develop between the Kelvin-Helmholtz vortices in a turbulent shear layer (Bernal and Roshko, 1986). This study is motivated by prior cavitation studies showing that the QSVs are the primary site for cavitation inception in high Reynolds number shear layers (Katz and O'Hern, 1986). The present shear layer is generated by a backward-facing step with height of $h=10 \mathrm{~mm}$, and the measurements have been performed at free-a stream velocity $(U)$ of 1.45 and $5.3 \mathrm{~m} / \mathrm{s}$, corresponding to a step-height based Reynolds number of $1.45 \times 10^{4}$ and $5.3 \times 10^{4}$ (Agarwal et al., 2018). The measurements have been 
14th International Symposium on Particle Image Velocimetry - ISPIV 2021

August 1-5, 2021

performed in a $12.5 \times 7.5 \times 4.5 \mathrm{~mm}^{3}$ sample volume where the cavitation inception events are most likely to occur along the core of 1-2 mm diameter QSVs. The sample volume is illustrated in Figure 1 (a). To perform statistical analysis of the QSV properties and pressure, the data is processed using the presently proposed pseudo-Lagrangian method that segregates the QSVs from the surrounding flow domain.

(a)

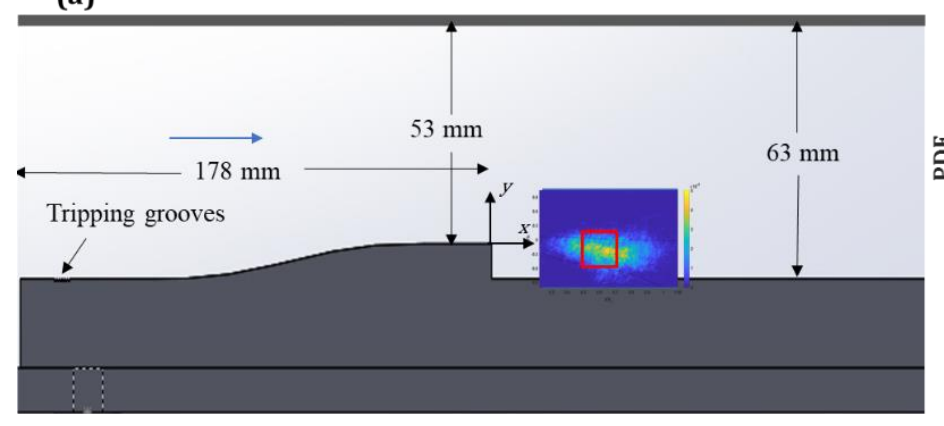

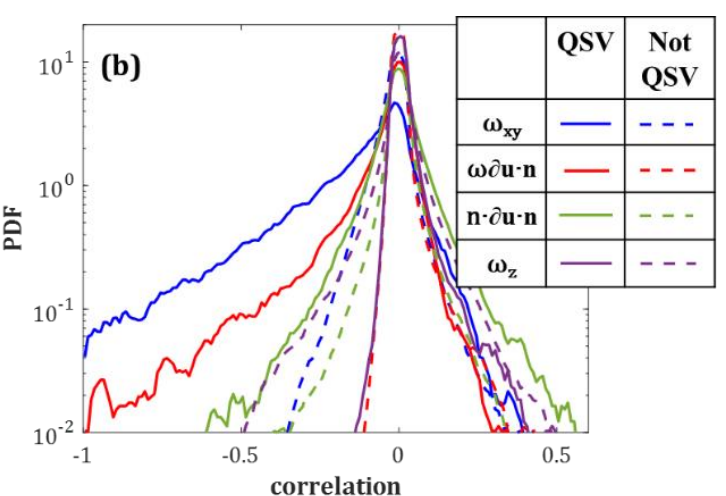

Figure 1: (a) The backward-facing step with a map of cavitation probability in the shear layer. The tomographic field of view is marked in red. (b) PDF of correlations of $\lambda_{2}$ with different variables used

\section{$2 \quad$ Method} in detecting the QSV.

The experiments have been performed in a small water tunnel fitted with a backward-facing step. The optical setup for the time-resolved tomographic PTV (Fig. 9b) involves four Pco.dimax cameras, arranged in the same horizontal $(\mathrm{x}, \mathrm{z}$ ) plane as the test section. The data are recorded at $14925 \mathrm{~Hz}$ with an image size of $624 \times 380$ pixels for $5.3 \mathrm{~m} / \mathrm{s}$, and at $7407 \mathrm{~Hz}$ with an image size of $1008 \times 596$ pixels for $1.45 \mathrm{~m} / \mathrm{s}$. In both cases, the spatial resolution is compromised to facilitate the high frame rates. The flow field is illuminated by a Photonics DM60-527 Nd:YLF laser, and the flow is seeded with $13 \mu \mathrm{m}$ diameter silver-coated hollow glass spheres. A total of 3500-5000 tracks are resolved in each instantaneous realization, with a typical distance between particles of $275 \mu \mathrm{m}$. The unstructured velocity and acceleration data from the particle tracks are interpolated using a Constrained Cost Minimization technique (Agarwal et al., 2021) to obtain structured data on velocity, material acceleration and their spatial gradients, at a grid resolution of $200 \mu \mathrm{m}$. The corresponding pressure distribution is obtained by spatially integrating the material acceleration using the 3D Omnidirectional method described in Wang et al. (2019).

A pseudo-Lagrangian detection method involving 95,000 synthetic particles is used to insure the spatial and temporal continuity of the detected structures. The particle motions are tracked using a fourth-order Runge Kutta method and cubic interpolation in space. The 3D velocity gradients are used for calculating the vorticity components $\omega_{\mathrm{i}}, \lambda_{2}$, and vortex stretching terms $\left(\omega_{i} \partial_{i} u_{j}\right)$. The QSV axis $n$ is identified as being perpendicular to the direction of spatial gradients of the vector sum of $\omega_{x}$ and $\omega_{y}$. To identify the QSVs and its evolution, the following parameters are utilized: (i) spanwise vorticity $\omega_{z}$, (ii) vorticity perpendicular to spanwise-direction, $\omega_{x y}$, (iii) $\lambda_{2}$, (iv) projection of vortex stretching term along the QSV axis $(\omega \cdot \partial \mathrm{u} \cdot \mathrm{n}),(\mathrm{v})$ projection of the strain rate tensor on the axis of the vortex $(n \cdot \partial u \cdot n)$, and (vi) the strain state parameter (Lund and Rogers, 1995). The position and the abovementioned six variables are recorded for each particle in five consecutive time steps, moving both forward and backward in time. For particles that are advected out of the volume $(\sim 10 \%$ of the 
total), only unidirectional time steps are considered. The resulting 45-dimensional dataset (9 variables at 5 times) is divided into 10 clusters using the correlations-based $k$-means method (Lloyd, 1982). Before clustering, the mean of each variable is removed and the quantities are normalized by their variance. The clusters with centers that have $\lambda_{2}$ lower than the mean, as well as $\omega_{x y}$ and vortex stretching magnitude higher than the mean, at all 5 times are chosen as QSV candidates. The detected QSV field is projected back to the grid using cubic interpolation. The grid-points that are have fewer than half of its neighbors classified as QSVs are re-labelled as not being QSV.

Figure 1(b) shows the probability density function of the correlation between $\lambda_{2}$ with the variables used for detecting the QSV, both within and outside of the QSVs. As is evident, within the QSV, $\lambda_{2}$ is more correlated with $\omega_{x y}$, axial vortex stretching, and axial strain rate, but is less correlated with $\omega_{z}$ compared to other regions (i.e. those not classified as QSVs). To visualize the high-dimensional data, t-distributed stochastic neighbor embedding (t-SNE, van der Maaten et al., 2008) is employed in Figure 2. This procedure reduces the data to two dimensions, while preserving the probability of being similar in higher dimensions. The maps are color coded by QSV detection, $\omega_{x y}, \lambda_{2}$ and pressure for one instance. As can be noted, the regions selected as QSVs are organized in clusters that typically have high $\omega_{x y}$, low $\lambda_{2}$ and low pressure. These clusters coincide with the points detected by the $k$ means algorithm.

(a) QSVs

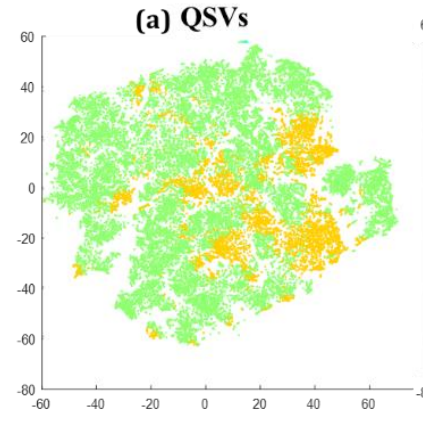

(b) $\omega_{x y}$

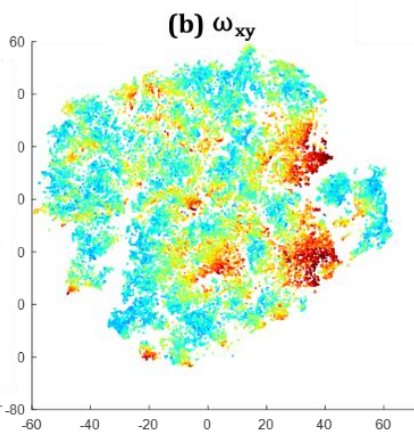

(c) $\lambda_{2}$

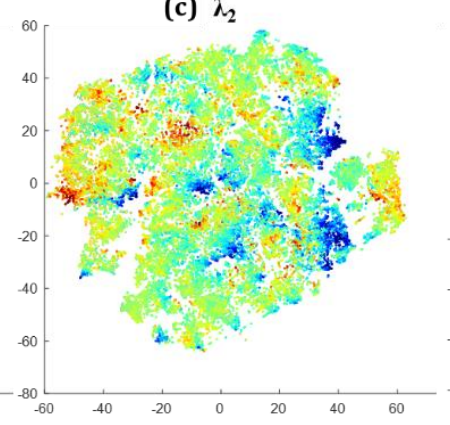

(d) Pressure

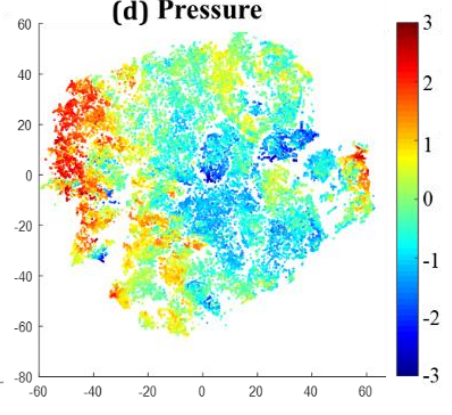

Figure 2: The t-SNE plots of the 45-dimensional matrix colored by: (a) detection of QSV based on the selected criteria, with 1.0 corresponding to QSVs, along with the corresponding mean-centered and variance-normalized values of: (b) vorticity in $x-y$ direction, (c) $\lambda_{2}$, and (d) pressure.

\section{$3 \quad$ Results}
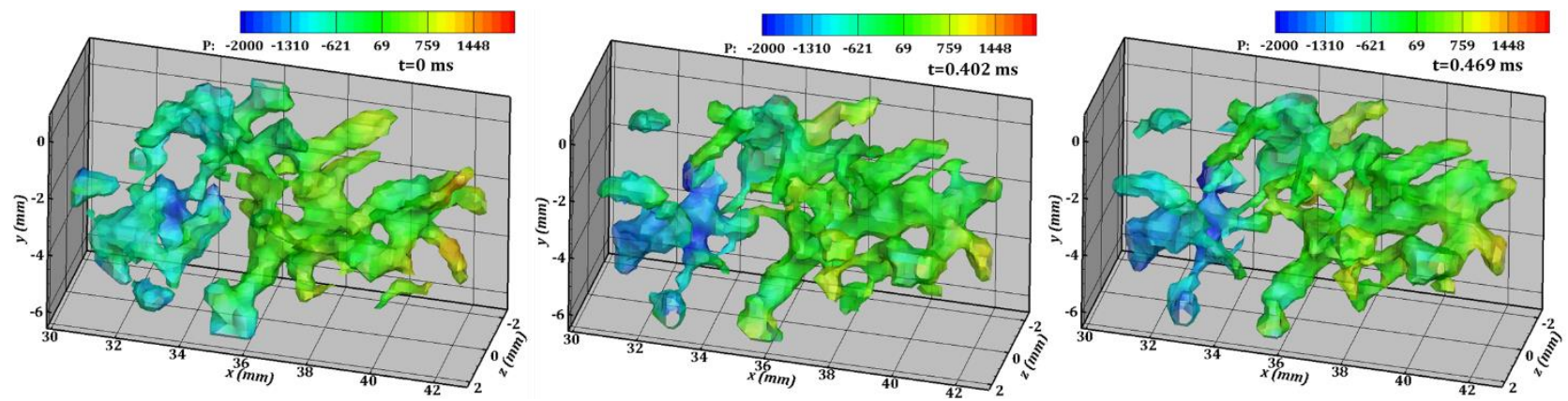

Figure 3: Samples of advected QSVs detected using the pseudo-Lagrangian method. The structures are color-coded with the pressure fluctuations in $\mathrm{Pa}$. 
14th International Symposium on Particle Image Velocimetry - ISPIV 2021

August 1-5, 2021

Figure 3 shows sample QSVs detected using the present method at the specified three times. The structures are evolving in time, while being convected at speeds of about $50 \%$ of the free-stream velocity. The structures are about 1-2 $\mathrm{mm}$ in diameter and over $5 \mathrm{~mm}$ in length, consistent with the size of the cavities. Statistical analysis of 28000 realizations for $5.3 \mathrm{~m} / \mathrm{s}$ and 15000 for $1.45 \mathrm{~m} / \mathrm{s}$ shows (Figure 4) that the pressure inside these structures is lower and the duration of pressure minima is longer than those in the surrounding fluid. The structures experience both high stretching $(\omega \cdot \partial u \cdot n$ $>0$, in Figure $4 \mathrm{c})$ and contraction $(\omega \cdot \partial \mathrm{u} \cdot \mathrm{n}<0)$. The Lagrangian correlations among variables are computed following the synthetic tracks, while recording the associated pressure, vorticity, stretching and the status of being either inside or outside of a QSV. Figure 5 shows the correlations of pressure at time $t+\Delta t$ with the vorticity (Fig. 5a), stretching (Fig. 5b) and contraction (Fig. 5c) for the same particle at time $t$, averaged over different instances and particles. Inside the QSVs, the pressure minima are more likely to occur in regions with high $\omega_{x y}$ at the same time $(\Delta t=0)$, follow (appear after) a stretching event $(\Delta t>0)$, and precede a contraction $(\Delta t<0)$. These trends are much weaker outside of the QSVs. These trends appear to be stronger for the lower velocity, presumably because of increase in turbulence level with increasing Reynolds number. Meanwhile, the Lagrangian pressure-pressure correlations (not shown) are higher for the higher velocity, with values of $\sim 0.3$ at $\Delta t U / h=1$ for $1.45 \mathrm{~m} / \mathrm{s}$ and $\sim 0.4$ for $5.3 \mathrm{~m} / \mathrm{s}$.

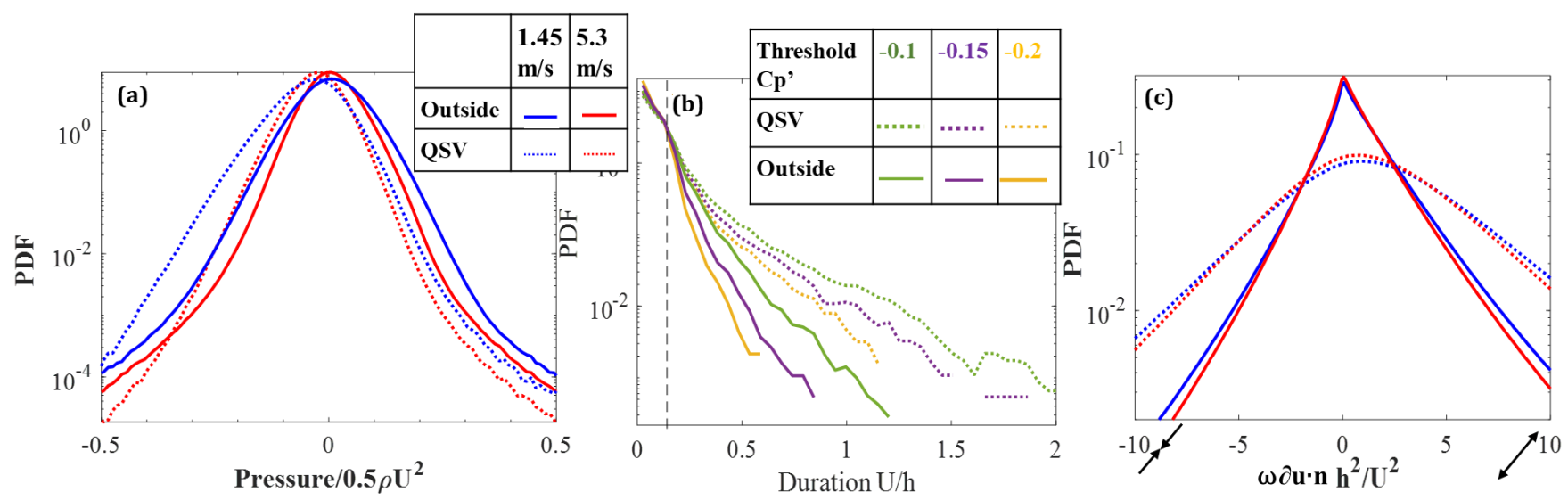

Figure 4: Probability density functions of: (a) pressure (b) durations of pressure lower than given thresholds and (c) vortex stretching term, inside and outside of the QSVs.
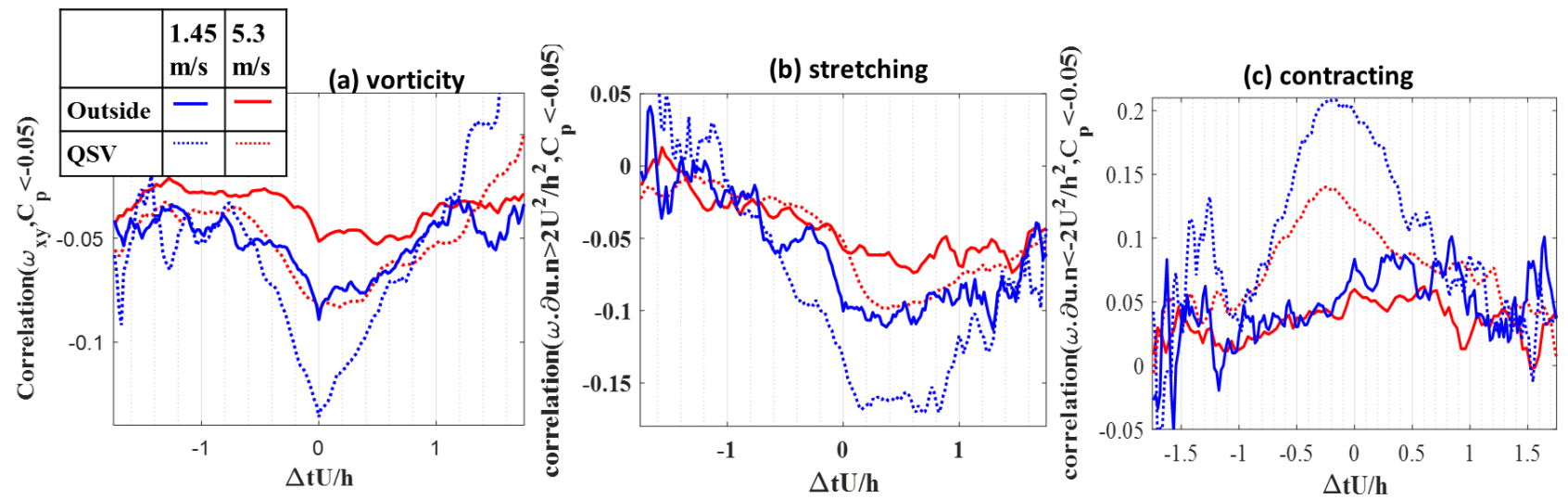

Figure 5: Lagrangian correlations of low-pressure events $\left(<-0.025 \rho U^{2}\right)$ at $t+\Delta t$ with vorticity, stretching $\left(>2 U^{2} / h^{2}\right)$ and contraction $\left(<-2 U^{2} / h^{2}\right)$ at time $t$. 
14th International Symposium on Particle Image Velocimetry - ISPIV 2021

August 1-5, 2021

\section{Conclusions}

A pseudo-Lagrangian vortex detection method is proposed that uses $k$-means clustering of particle positions and variables associated with the velocity gradients to detect secondary quasi streamwise vortices in the near field of a turbulent shear layer. Results show that regions with pressure minima are more likely to be located within the QSVs than outside of them. These low-pressure events are more likely to occur after periods of stretching with duration of $\Delta t U / h \sim 1$ and before contraction events. Ongoing analysis focuses on characterizing the structure of these vortices, for example, how the pressure is distributed along their axes and for how long. These data could then be used for predicting the rate of cavitation inception events.

\section{Acknowledgement}

This project has been supported in part by ONR MURI grant: Predicting Turbulent Multi-Phase Flows with High Fidelity - A Physics-Based Approach, and in part by ONR grant No. N00014-18-1-2635.

\section{References}

Adrian R.J., Christensen K.T., and Liu Z.C.(2000) Analysis and interpretation of instantaneous turbulent velocity fields, Exp. Fluids 29, pp. 275-290.

Agarwal, K., Ram, O. and Katz, J. (2018) Cavitating structures at inception in turbulent shear flow. In Proceedings for 10th International Symposium on Cavitation, Baltimore, USA.

Agarwal, K., Ram, O., Wang, J., Lu, Y., \& Katz, J. (2021). Reconstructing velocity and pressure from noisy sparse particle tracks using constrained cost minimization. Experiments in Fluids, 62(4), 1-20.

Bernal, L. P., \& Roshko, A. (1986). Streamwise vortex structure in plane mixing layers. Journal of Fluid Mechanics, 170, 499-525.

Haller, G., \& Sapsis, T. (2011). Lagrangian coherent structures and the smallest finite-time Lyapunov exponent. Chaos: An Interdisciplinary Journal of Nonlinear Science, 21(2), 023115.

Hunt J. C. R., Way A., Moin P.(1998) Eddies, stream, and convergence zones in turbulent flows. Center for Turbulence Research Report CTR-S88, Standford University, 1, 5.

Jeong J., Hussain F. (1995) On the identification of a vortex. Journal of Fluid Mechanics 285, 1, 69-94. 1,5 .

Katz, J., \& O’hern, T. J. (1986). Cavitation in large scale shear flows. Journal of fluids engineering.

Lund, T. S., \& Rogers, M. M. (1994). An improved measure of strain state probability in turbulent flows. Physics of Fluids, 6(5), 1838-1847.

Lloyd, S. (1982). Least squares quantization in PCM. IEEE transactions on information theory, 28(2), 129-137.

Schanz, D., Gesemann, S., \& Schröder, A. (2016) Shake-The-Box: Lagrangian particle tracking at high particle image densities. Experiments in fluids, 57(5), 70.

Schlueter-Kuck, K. L., \& Dabiri, J. O. (2017). Identification of individual coherent sets associated with flow trajectories using coherent structure coloring. Chaos: An Interdisciplinary Journal of Nonlinear Science, 27(9), 091101. 
14th International Symposium on Particle Image Velocimetry - ISPIV 2021

August 1-5, 2021

van der Maaten, L.J.P.; Hinton, G.E. (2008). Visualizing Data Using t-SNE. Journal of Machine Learning Research. 9: 2579-2605.

Wang, J., Zhang, C., Katz, J. (2019) GPU-based, parallel-line, omni-directional integration of measured pressure gradient field to obtain the 3D pressure distribution. Experiments in Fluids, 60(4), p.58. 